\title{
Markov Random Field driven Region-Based Active Contour Model (MaRACel): Application to Medical Image Segmentation
}

\author{
Jun $\mathrm{Xu}^{\star}$, James P. Monaco*, and Anant Madabhushi \\ Department of Biomedical Engineering, Rutgers University, USA \\ \{junxu, jpmonaco, anantm\}@rci.rutgers.edu
}

\begin{abstract}
In this paper we present a Markov random field (MRF) driven region-based active contour model (MaRACel) for medical image segmentation. State-of-the-art region-based active contour (RAC) models assume that every spatial location in the image is statistically independent of the others, thereby ignoring valuable contextual information. To address this shortcoming we incorporate a MRF prior into the AC model, further generalizing Chan \& Vese's (CV) and Rousson and Deriche's (RD) AC models. This incorporation requires a Markov prior that is consistent with the continuous variational framework characteristic of active contours; consequently, we introduce a continuous analogue to the discrete Potts model. To demonstrate the effectiveness of MaRACel, we compare its performance to those of the CV and RD AC models in the following scenarios: (1) the qualitative segmentation of a cancerous lesion in a breast DCE-MR image and (2) the qualitative and quantitative segmentations of prostatic acini (glands) in 200 histopathology images. Across the 200 prostate needle core biopsy histology images, MaRACel yielded an average sensitivity, specificity, and positive predictive value of $71 \%, 95 \%, 74 \%$ with respect to the segmented gland boundaries; the $\mathrm{CV}$ and $\mathrm{RD}$ models have corresponding values of $19 \%, 81 \%, 20 \%$ and $53 \%, 88 \%, 56 \%$, respectively.
\end{abstract}

Keywords: Segmentation, medical imaging, prostate cancer, breast cancer, MRI, digital pathology.

\section{Introduction}

An active contour (AC) model performs segmentation by evolving a curve towards the minimum of an energy functional. Based on the type of image information driving the model, an AC may be categorized as either boundary-based [1] or region-based [2]. Region-based AC (RAC) models (the type of model considered in this work) employ statistical information derived from the segmented regions to drive the AC. Additionally, RACs employ level sets [3] instead of parameterized contours [2, 4]. The attraction of level sets lies in their ability to

\footnotetext{
^ J. Xu and J. Monaco are joint first authors.
}

T. Jiang et al. (Eds.): MICCAI 2010, Part III, LNCS 6363, pp. 197 204, 2010.
(C) Springer-Verlag Berlin Heidelberg 2010 
implicitly handle topological changes such as boundary splitting and merging. An early RAC model, called the region competition model [5], used generalized Bayes and minimum description length criteria to formulate the curve evolution function. Inspired by the Mumford-Shah functional, Chan \& Vese's (CV) [2] and Rousson and Deriche's (RD) [4] RAC models used statistics derived from the intensities of each region to drive their ACs.

The shortcoming of most RAC models lies in their assumption that each spatial location in the image is statistically independent of the others. Unfortunately, this assumption does not hold for most (or perhaps any) real images. To address this assumption, we propose the integration of Markov random fields (MRFs) into the RACs. MRFs [6] provide an effective and tractable (via the Markov property) means for modeling spatial information within a Bayesian framework 6].

The contribution of this paper is an MRF-driven RAC model (MaRACel). Specifically, MaRACel incorporates a Markov prior into the RD model [4. Intuitively, the effect of integrating this prior is similar to performing a probabilistic morphological close operation: edges randomly occurring (due to noise) in the regions are much less likely to result in spurious boundaries. For the Markov prior we introduce a continuous analogue of the discrete Potts model. This continuous extension is needed to ensure compatibility with variational calculus, the foundation of AC models. To demonstrate the superiority of MaRACel over the popular $\mathrm{CV}$ and RD models we qualitatively and quantitatively compare their performances in the task of segmenting prostatic acini (glands) in 200 digitized images of core needle biopsies.

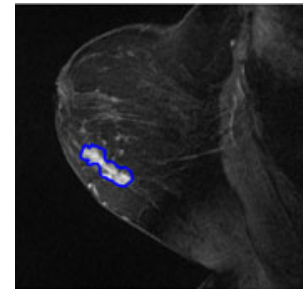

(a)

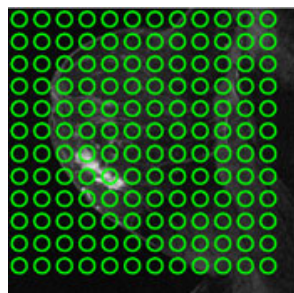

(b)

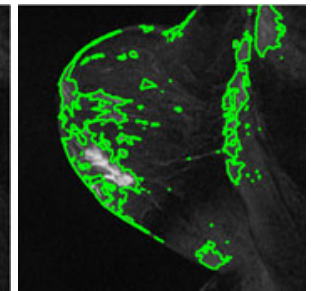

(c)

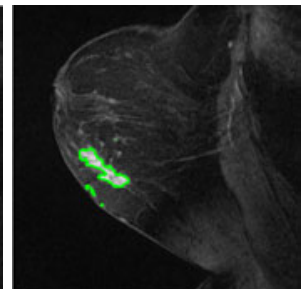

(d)

Fig. 1. Qualitative segmentation results for tumor boundary segmentation for breast DCE-MR image. (a) Single slice of DCE-MR image with manual segmentation of tumor region (in blue); segmentation results (in green) for (b) CV model, (c) RD model and (d) MaRACel.

To better highlight the need for MaRACel, we provide an illustrative example (Figure 1) of the automated detection of a lesion on a dynamic contrast enhanced (DCE) breast MR image [7]. The segmentation results for the RD and $\mathrm{CV}$ models, shown in columns (b) and (c), reveal the failure of these models to eliminate spurious noisy regions. (Note that $\mathrm{CV}$ model performs so poorly that the model does not evolve beyond its initial contours.) This is a result of the 
RD and CV models' inability to account for the fact that spatially proximate pixels are more likely to belong to the same regions. As evinced in column (d), this issue can be rectified by incorporating a Markov prior — such as the Potts model - into the AC, thereby encouraging neighboring pixels to share the same class (i.e. be included in the same segmented region).

The remainder of the paper proceeds as follows: Section 2 establishes the statistical model for describing random images and introduces the MRF prior. Section 3 discusses how the curve evolution function can be derived from this statistical model. In Section 4 we apply MaRACel to the segmentation of prostatic glands, qualitatively and quantitatively evaluating its segmentation performance. Finally, we offer concluding remarks in Section 5 .

\section{Statistical Framework for the MaRACel Model}

Let $\mathcal{C}=(C, \mathbf{f})$ define a color image, where $C \subset \mathbb{R}^{2}$ establishes the image region and f : $C \rightarrow \mathbb{R}^{3}$ reflects the intensities of the three color channels. Let $x: C \rightarrow\left\{\Lambda_{1}, \Lambda_{2}\right\}$ be a function mapping each point in $C$ to one of two discrete classes $\left\{\Lambda_{1}, \Lambda_{2}\right\}$. Thus $x$ segments the image into regions $C_{1}$ and $C_{2}$ (e.g. the foreground and the background), where $C_{1} \cup C_{2}=C$ and $c \in C_{i}$ implies $x_{c}=\Lambda_{i}$.

The goal is to determine the function $x$ that best segments the region $C$ given the observed colors $\mathbf{f}$. Since we use a Bayesian framework, we consider the functions $\mathbf{f}$ and $x$ as observations of the random processes $\mathbf{F}$ and $X$. Furthermore, the optimal segmentation (with respect to probability of error) is given by the maximum a posteriori (MAP) estimate [8]:

$$
\max _{x} P(X=x \mid \mathbf{F}=\mathbf{f}) \propto \max _{x} P(\mathbf{F}=\mathbf{f} \mid X=x) P(X=x)
$$

Thus, maximizing the a posteriori probability $P(X=x \mid \mathbf{F}=\mathbf{f})$ is equivalent to maximizing the product of the conditional $P(\mathbf{F}=\mathbf{f} \mid X=x)$ and prior $P(X=x)$ probabilities.

Note that $P(X=x)$, which signifies the probability of the event $\left\{X_{c}=x_{c}, \forall c \in\right.$ $C$ \}, should not be confused with $P\left(X_{c}=x_{c}\right)$, which indicates the probability of the event $\left\{X_{c}=x_{c}\right\}$ at $c$. Additionally, when it does not cause ambiguity we will henceforth omit the random variables from the probability functions, e.g. $P(x) \equiv P(X=x)$.

\subsection{Estimating the Prior Probability}

To simplify the prior probability we invoke the Markov property: $P\left(x_{c} \mid x_{-c}\right)=$ $P\left(x_{c} \mid x_{\eta_{c}}\right)$, where $x_{-c}$ abbreviates $\{x(r): r \in C, r \neq c\}, x_{\eta_{c}}$ abbreviates $\{x(r): r \in$ $\left.\eta_{c}, r \neq c\right\}$, and the neighborhood $\eta_{c}$ of $c$ is any bounded region such that $s \in$ $\eta_{c} \leftrightarrow c \in \eta_{s}, c \notin \eta_{c}$, and $c, s \in C$. Using these simplified conditional probabilities, the prior probability of the event $\left\{X_{c}=x_{c}, \forall c \in C\right\}$ can be approximated by the normalized pseudo-likelihood [9] as follows:

$$
P(X=x) \approx \frac{1}{Z} \prod_{i=1}^{2} \prod_{c \in C_{i}} P\left(X_{c}=\Lambda_{i} \mid x_{\eta_{c}}\right)^{d c}
$$


where $Z$ ensures summation to one and $d c$ represents the bin volume which guarantees the correct continuum limit [10. We choose to model each of the conditional distributions in (2) using a continuous analogue of the Potts model [11:

$$
P\left(X_{c}=\Lambda_{i} \mid x_{\eta_{c}}\right)=\frac{1}{Z_{c}} \exp \left\{-\lambda \int_{\eta_{c} \cap C_{i}} d c\right\}=\frac{1}{Z_{c}} \exp \left\{-\lambda A\left(\eta_{c}, \Lambda_{i}\right)\right\},
$$

where $Z_{c}$ is a normalizing constant, $A\left(\eta_{c}, C_{i}\right)$ signifies the area of the region defined by $\eta_{c} \cap C_{i}$, and $\lambda \in \mathbb{R}$ is an appropriately selected constant. Note that the greater the value of $\lambda$, the greater the tendency for neighboring points in the MAP estimate to belong to the same class.

\subsection{Estimating the Conditional Probability}

We next estimate the conditional probability $P(\mathbf{f} \mid x)$. Since each vector $\mathbf{F}_{c}$ given $\left\{X_{c}=x_{c}\right\}$ is assumed conditionally independent of all other $\mathbf{F}_{c}, P(\mathbf{f} \mid x)$ can be expressed as follows:

$$
P(\mathbf{f} \mid x)=\prod_{i=1}^{2} \prod_{c \in C_{i}} P\left(\mathbf{f}_{c} \mid X_{c}=\Lambda_{i}\right)^{d c},
$$

where $d c$ again assures the correct continuum limit [10.

Similar to [5, 4], we assume each conditional probability $P\left(\mathbf{f}_{c} \mid X_{c}=\Lambda_{i}\right)$ is distributed normally with the following mean $\mu_{i}$ and covariance $\Sigma_{i}$ :

$$
\mu_{i}=\frac{1}{\left|C_{i}\right|} \int_{C_{i}} \mathbf{f}_{c} d c \quad \text { and } \quad \Sigma_{i}=\frac{1}{\left|C_{i}\right|} \int_{C_{i}}\left(\mathbf{f}_{c}-\mu_{i}\right)\left(\mathbf{f}_{c}-\mu_{i}\right)^{T} d c .
$$

where $\left|C_{i}\right|=\int_{C_{i}} d c$ is the area of region $C_{i}$.

\section{Variational Framework for the MaRACel Model}

\subsection{Energy Functional of MaRACel Model}

We now wish to perform MAP estimation within the active contour framework. We begin by using Equations (11), (2), and (4) to express the log of the a posteriori probability - whose maximum will correspond to that of the a posteriori probability - in integral form:

$$
\begin{aligned}
\log P(x \mid \mathbf{f}) & =\log P(\mathbf{f} \mid x)+\log P(x) \\
& =\sum_{i=1}^{2} \int_{C_{i}} \log P\left(\mathbf{f}_{c} \mid X_{c}=\Lambda_{i}\right)+\log P\left(X_{c} \approx \Lambda_{i} \mid x_{\eta_{c}}\right) d c-\log (Z) .
\end{aligned}
$$


This formulation can be rewritten as an energy functional [2]:

$$
\begin{aligned}
E(\phi) & =-\int_{C}\left\{\alpha\left[H(\phi) \log P\left(\mathbf{f}_{c} \mid X_{c}=\Lambda_{1}\right)+(1-H(\phi)) \log P\left(\mathbf{f}_{c} \mid X_{c}=\Lambda_{2}\right)\right]\right. \\
& +\beta\left[H(\phi) \log P\left(X_{c} \approx \Lambda_{1} \mid x_{\eta_{c}}\right)+(1-H(\phi)) \log P\left(X_{c} \approx \Lambda_{2} \mid x_{\eta_{c}}\right)\right] \\
& -\gamma|\nabla H(\phi)|\} d c,
\end{aligned}
$$

where $H(\cdot)$ is the Heaviside function and $\phi: C \rightarrow \mathbb{R}$ is a level set function such that $\phi(c)>0$ if $c \in C_{1}$ and $\phi(c)<0$ if $c \in C_{2}$. Thus, the $\phi$ that minimizes $E(\phi)$ establishes the boundary that corresponds to the MAP estimate (under the pseudo-likelihood approximation in (2) ). Note that the final term $\gamma|\nabla H(\phi)|$ in (77) results from incorporating the boundary length for the purpose of regularization; this term was not derived from (6), and thus the minimizer of (7) will not precisely correspond to the MAP estimate.

\subsection{Curve Evolution Functional of MaRACel Model}

Applying the Euler-Lagrange equations to (7) and then substituting in (3) and (5) yields the curve evolution function for MaRACel

$$
\begin{aligned}
\frac{\partial \phi}{\partial t}= & \delta(\phi)\left\{\alpha \left[\left(\mathbf{f}_{c}-\mu_{2}\right)^{T} \Sigma_{2}^{-1}\left(\mathbf{f}_{c}-\mu_{2}\right)-\left(\mathbf{f}_{c}-\mu_{1}\right)^{T} \Sigma_{1}^{-1}\left(\mathbf{f}_{c}-\mu_{1}\right)\right.\right. \\
& \left.\left.+\log \frac{\left|\Sigma_{2}\right|}{\left|\Sigma_{1}\right|}\right]+\beta\left(A\left(\eta_{c}, C_{1}\right)-A\left(\eta_{c}, C_{2}\right)\right)+\gamma \operatorname{div}\left(\frac{\nabla \phi}{\|\nabla \phi\|}\right)\right\},
\end{aligned}
$$

where $\beta=\alpha \cdot \lambda$. Note that the relationship between MaRACel and the RD and CV models is as follows: Setting $\beta=0$ in the MaRACel evolution function (8) reduces it to the $\mathrm{RD}$ model. If we further stipulate that $\Sigma_{1}$ and $\Sigma_{2}$ are both identity matrices, the RD model devolves into the $\mathrm{CV}$ model.

\section{Experimental Results and Discussion}

To illustrate the effectiveness of our proposed model, we now qualitatively and quantitatively compare the gland segmentation performance of MaRACel to those of the $\mathrm{CV}$ and $\mathrm{RD}$ models. Note that since cancerous and benign glands have unique morphologies, the ability to accurately delineate their boundaries is essential in both automated cancer detection [11] and Gleason grading (i.e. the stratification of prostate cancer by aggressiveness).

\subsection{Model Parameters and Data Description}

The initial contours for the CV, RD, and MaRACel models are given by an array of circles dispersed regularly across the image. (These circles were unintentionally depicted by the poor segmentation results in Figure 1(b).) Empirically, we found 
that we achieve more robust performance if the MRF weighting term $\beta$ in (8) begins at zero and then increases with each iteration (i.e. as the segmentations become more accurate) before leveling off. Thus $\beta$ is determined as follows: $\beta=\frac{2 \beta_{0}}{\pi} \arctan [0.2(t-1)]$, where $t$ is the time in iterations. The values for $\alpha, \beta_{0}$ and $\gamma$ in (8) are 5, 0.1 and 15, respectively. The neighborhood $\eta_{c}$ is a $21 \times 21$ window centered at pixel $c$ (and excluding $c$ ). Thus the area $A\left(\eta_{c}, \Omega_{i}\right)$ in (8) is simply the number of pixels with class $\Lambda_{i}$ that lie within the window $\eta_{c}$ (excluding the center pixel $c$ ).

The dataset includes 200 images obtained from Hematoxylin \& Eosin (H\&E) stained prostate biopsy samples digitized at 20x optical magnification using an Aperio whole-slide digital scanner. Each image includes one or more prostatic glands. The glands in each of the 200 images were manually segmented (to provide ground truth for quantitative evaluation).

\subsection{Qualitative Results}

The goal is to accurately delineate the boundaries of the glandular lumens for each image in column (a) of Figure 2. The segmentation for the RD and CV models, shown in columns (c) and (d) in Figure 2, reveal the inability of these models to eliminate the small, spurious regions that appear due to noise. That is, the $\mathrm{CV}$ and RD models find background regions within the glands. The segmentation results for MaRACel are given in column (d). MaRACel removes these false regions, yielding a single segmented region for each glandular structure.

\subsection{Quantitative Results}

For each image the set of pixels lying within the manual delineations of the glands is denoted as $\mathcal{A}(G)$. The set of pixels lying within any boundary resulting from the specified AC model is denoted as $\mathcal{A}(S)$. The sensitivity (SN), specificity $(\mathrm{SP})$, and positive predictive value $(\mathrm{PPV})$ are then defined as follows: $\mathrm{SN}=$ $\frac{|\mathcal{A}(S) \cap \mathcal{A}(G)|}{|\mathcal{A}(G)|}, \mathrm{SP}=\frac{|C|-|\mathcal{A}(S) \cup \mathcal{A}(G)|}{|C|-|\mathcal{A}(G)|}$, and PPV $=\frac{|\mathcal{A}(S) \cap \mathcal{A}(G)|}{|\mathcal{A}(S)|}$, where $|\cdot|$ denotes the cardinality of the set. We compute SN, SP and PPV for each image, and then determine the average and standard deviation across the 200 images. These statistics are reported in Table 1 for the CV, RD and MaRACel models.

Table 1. Quantitative evaluation of segmentation results for the CV, RD and MaRACel models. The average and standard deviation of the SN, SP and PPV over the 200 histopathology images are provided.

\begin{tabular}{|c|c|c|c|}
\hline & Sensitivity $(S N)$ & Specificity $(S P)$ & Positive Predictive Value $(P P V)$ \\
\hline CV & $0.19 \pm 0.06$ & $0.81 \pm 0.01$ & $0.20 \pm 0.06$ \\
\hline RD & $0.53 \pm 0.10$ & $0.88 \pm 0.11$ & $0.56 \pm 0.11$ \\
\hline MaRACel & $\mathbf{0 . 7 1} \pm 0.10$ & $\mathbf{0 . 9 5} \pm 0.01$ & $\mathbf{0 . 7 4} \pm 0.10$ \\
\hline
\end{tabular}



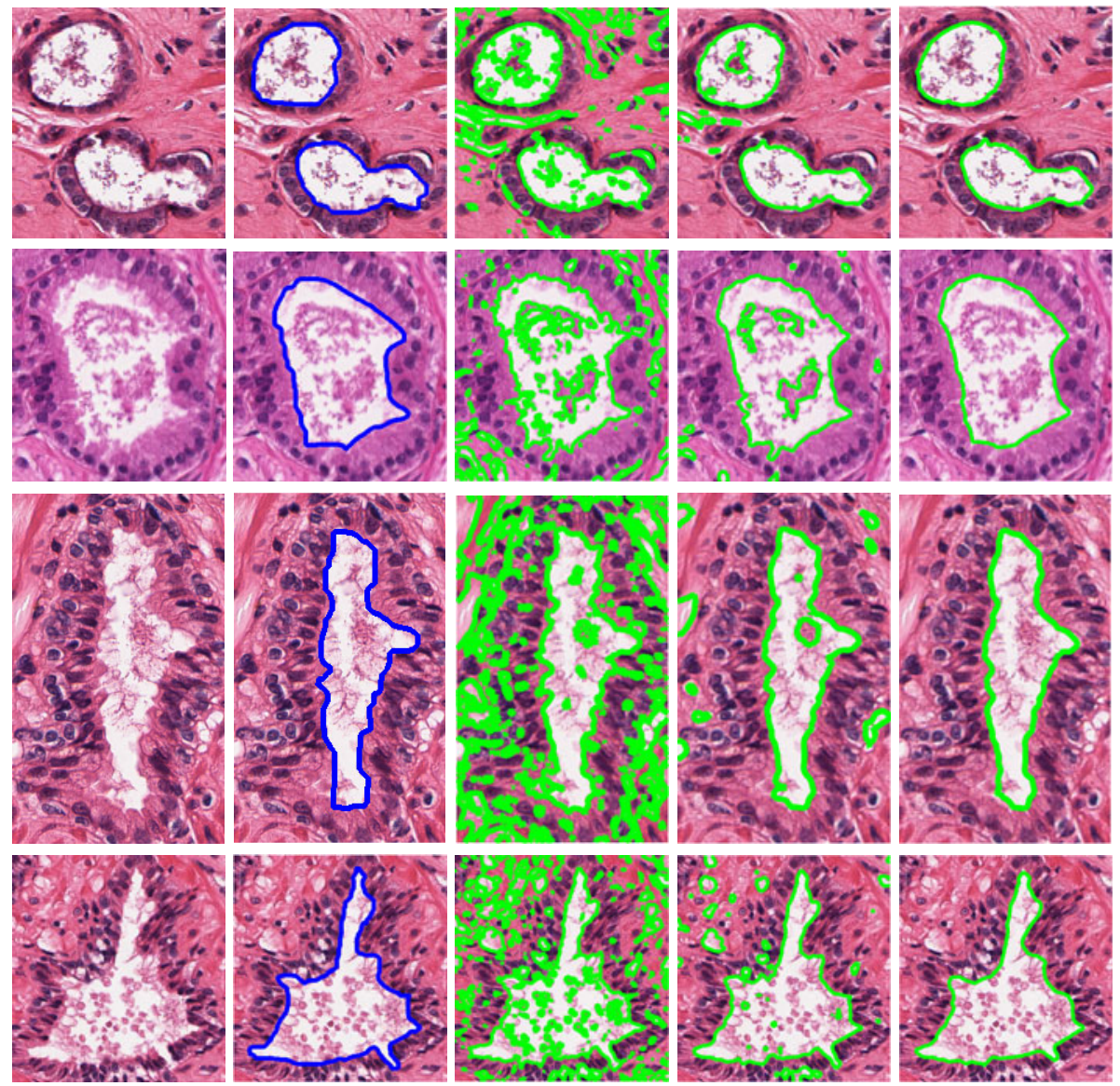

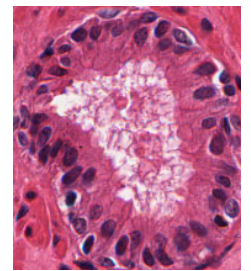

(a)

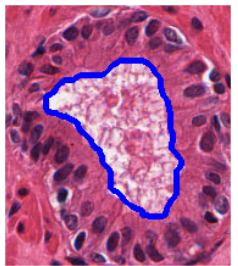

(b)

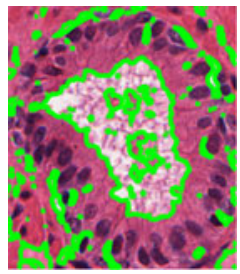

(c)

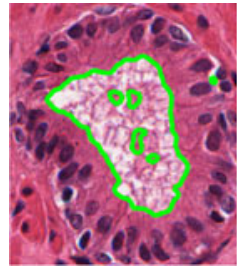

(d)

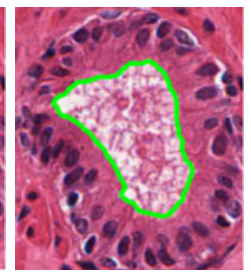

(e)

Fig. 2. Qualitative segmentation results for prostatic glands in digitized biopsy samples. (a) original images; (b) manual segmentations of the glandular boundaries (in blue); segmentation results (in green) for (c) CV model, (d) RD model and (e) MaRACel.

\section{Concluding Remarks}

In this paper we presented MaRACel, a generalization of Chan \& Vese's and Rousson and Deriche's active contour models, that incorporates Markov random 
fields into the AC. Currently, the region-based AC models only extract global statistical information and ignore valuable local contextual information. Incorporating an MRF prior into the AC provides a means for modeling this contextual information which is so essential in rejecting spurious edges and other forms of noise. To validate our model we quantitatively and qualitatively compared MaRACel with Chan \& Vese's and Rousson and Deriche's AC models in the task of identifying glandular boundaries in prostate histopathology images. MaRACel significantly outperformed the other models in terms of average sensitivity, specificity, and positive predictive value.

\section{Acknowledgments}

This work is supported by NIH Grant Nos. R01-CA-136535-01, R21-CA-12718601, and R03-CA-128081-01, the NJ Commission on Cancer Research, the Coulter Foundation, the Cancer Institute of NJ, and Bioimagene Inc.

\section{References}

1. Caselles, V., Kimmel, R., Sapiro, G.: Geodesic active contours. IJCV 22(1), 61-79 (1997)

2. Chan, T.F., Vese, L.A.: Active contours without edges. IEEE TIP 10(2), 266-277 (2001)

3. Sethian, J.A.: A fast marching level set method for monotonically advancing fronts. Proceedings of the National Academy of Sciences of the United States of America 93(4), 1591-1595 (1996)

4. Rousson, M., Deriche, R.: A variational framework for active and adaptative segmentation of vector valued images, pp. 56-61 (2002)

5. Zhu, S., Yuille, A.: Region competition: unifying snakes, region growing, and bayes/mdl for multiband image segmentation. IEEE TPAMI 18(9), 884-900 (1996)

6. Geman, S., Geman, D.: Stochastic relaxation, gibbs distributions, and the bayesian restoration of images. IEEE TPAMI 6(6), 721-741 (1984)

7. Agner, S., Soman, S., Libfeld, E., McDonald, M., Thomas, K., Englander, S., Rosen, M., Chin, D., Nosher, J., Madabhushi, A.: Textural kinetics: A novel dynamic contrast-enhanced (dce)-mri feature for breast lesion classification. Journal of Digital Imaging, May 28 (2010) [Epub. ahead of print]

8. Paragios, N., Deriche, R.: Geodesic active regions and level set methods for supervised texture segmentation. IJCV 46(3), 223-247 (2002)

9. Besag, J.: Statistical analysis of non-lattice data. Journal of the Royal Statistical Society, Series D (The Statistician) 24(3), 179-195 (1975)

10. Cremers, D., Mikael, R., Rachid, D.: A review of statistical approaches to level set segmentation: Integrating color, texture, motion and shape. IJCV 72, 195-215 (2007)

11. Monaco, J.P., Tomaszewski, J.E., Feldman, M.D., Hagemann, I., Moradi, M., Mousavi, P., Boag, A., Davidson, C., Abolmaesumi, P., Madabhushi, A.: Highthroughput detection of prostate cancer in histological sections using probabilistic pairwise markov models. Medical Image Analysis 14(4), 617 (2010) 